\title{
A study on evaluation of knee osteoarthritis with MRI and comparing it with CT scan, high resolution USG and conventional radiography
}

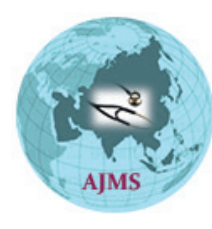

\author{
Raju Singha', Chanchal Kumar Dalai², Deblina Sarkar ${ }^{3}$ \\ ${ }^{1}$ Assistant Professor, Department of Radio-diagnosis, ${ }^{2}$ Associate Professor, Department of Pharmacology, ${ }^{3}$ Assistant \\ Professor, Department of Community Medicine, College of Medicine and JNM Hospital, The West Bengal University of \\ Health Sciences, Kalyani, West Bengal, India
}

Background: Knee osteo-arthritis is widely prevalent in the elderly population in our society and associated with significant morbidity and poor quality of life. Early diagnosis of the condition can enable timely and proper care for the patients. Magnetic Resonance Imaging, CT Scan, Ultrasonography and plain radiography are the different modalities of imaging that are commonly used for detection and diagnosis of knee osteo-arthritis. Aims and Objectives: To find out the early osteoarthritic changes of knee by Magnetic Resonance Imaging and compare those findings with conventional radiography, high frequency USG and CT scan findings. Materials and Methods: Patients suffering from knee osteoarthritis (OA) as per American College of Rheumatology guideline criteria $(n=56)$ underwent imaging of the knee using plain radiography, ultrasonography, CT scan and MRI. The imaging findings studied in the patients were joint space narrowing (JSN), meniscal abnormality, Baker's cyst, cruciate ligament abnormality, knee effusion, subchondral cyst, and loose bodies. A comparison between radiography, CT scan and USG was done for the imaging findings with MRI as the reference standard. Z-test of proportionality was used to find statistically significant difference for the three imaging modalities. A $P<0.05$ was deemed statistically significant. Results: The mean age of the patients was 61 years ( 38 males). The tibiofemoral compartment was most commonly affected. CT scan was more sensitive than radiography in detecting subchondral cyst $(P=0.018)$ and loose bodies $(P=0.004)$. USG and MRI were equally sensitive in detecting knee effusion $(P=0.22)$ and synovial thickening $(P=0.10)$. CT scan and MRI were equally sensitive in detecting subchondral cyst $(P=1.00)$ and loose bodies $(P=0.22)$. Conclusion: While CT imaging was more sensitive for detection of subchondral cysts and loose bodies than conventional radiography, it was as sensitive as MRI in detecting these findings in the study group. Additional study is warranted to assess diagnostic performance of CT scan and MRI in the diagnosis and progression of knee OA.

Key words: Knee osteoarthritis; Magnetic resonance imaging; Ultrasonography; Radiography

\section{INTRODUCTION}

Osteoarthritis (OA) is a widely prevalent musculoskeletal disease in Indian adults, resulting in significant morbidity in life. The disease is also a considerable economic burden on the health care resources of the country. ${ }^{1}$ The knee is the weight-bearing joint of the body and most commonly

\section{Access this article online}

Website:

http://nepjol.info/index.php/AJMS DOI: 10.3126/ajms.v12i12.39174

E-ISSN: 2091-0576

P-ISSN: 2467-9100

Copyright (c) 2021 Asian Journal of Medical Sciences

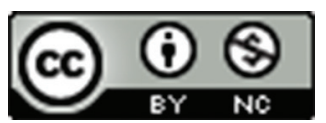

This work is licensed under a Creative Commons Attribution-NonCommercial 4.0 International License. 
causing discrepancies in interpretation. For example, subchondral cysts are a common finding in knee OA, but plain radiographs often fail to detect this feature. Similarly, osteophytes may be missed in plain radiography resulting in significant delay in diagnosis and therefore initiation of treatment. MRI and CT scan are newer imaging modalities which have considerable higher sensitivity and specificity as compared to plain radiography. It also allows better visualization of the tibiofemoral (TF) and patella-femoral compartments of the knee joint. Ultrasonography of the knee joint cavity is also used to ascertain the thickening of the synovial membrane and presence of knee effusion. ${ }^{4}$ In the present study, we have studied the imaging features of knee osteo-arthritis using all the four imaging modalities. We compared the finding in knee OA between plain radiography and CT scan with MRI as the reference standard.

\section{Aims and objectives}

To find out the early osteoarthritic changes of knee by and compare those findings with conventional radiography, high frequency USG and CT scan findings.

\section{MATERIALS AND METHODS}

\section{Study design and ethical approval}

The study design is a cross-sectional descriptive study conducted in the Department of Radiodiagnosis and Department of Physical Medicine and Rehabilitation of North Bengal Medical College and Hospital for a period of 1 year. A topic was selected with the title of "A study on evaluation of knee OA with MRI and comparing it with CT scan, high resolution USG and conventional radiography" and first a protocol was formed, and it was submitted to the institutional ethics committee for ethical permission. After ethical permission, a proforma was made and it was pretested and then a final proforma prepared. After taking informed consent, the patients with knee pain diagnosed clinically as $\mathrm{OA}$ and referred to the department of Radiodiagnosis for imaging were examined using proper ACR clinical guideline. Then, these screened patients, who fulfill the inclusion and exclusion criteria, were evaluated with MRI, followed by conventional radiography, high frequency USG and CT scan.

\section{Study population}

This study was conducted among the patients who were referred to the Department of Radiodiagnosis for imaging with clinical features of knee OA fulfilling inclusion and exclusion criteria.

\section{Inclusion criteria}

Clinical features of knee OA as per American College of Rheumatology (ACR) guideline as mentioned afterwards.

\section{Exclusion criteria}

a) Patients with knee pain not fulfilling ACR guideline criteria.

b) Patients with specific medical and/or surgical conditions which may involve the knee joint, such as tumor, recent injury, septic arthritis, and operated knee.

c) Patients suffering from other forms of arthritis such as Rheumatoid arthritis, seronegative arthritis, and depositional arthritis.

d) Patients with contraindication for doing MRI.

e) Patients not giving consent for study.

\section{Clinical features for diagnosis of knee OA las per ACR guideline)}

a) Knee joint pain, with 3 other criteria among mentioned criteria below.

b) Age: $>50$.

c) Morning stiffness lasting for $<30 \mathrm{~min}$.

d) Crepitation or grating sensation on active movement.

e) Bony tenderness of knee joint.

f) Palpable bony enlargement around knee joint.

g) No detectable warmth of the joint to touch.

Sample size and sampling technique

Total 56 patients were studied using purposive sampling technique for selection of the patients

\section{Study tools}

a) Predesigned, Pretested, Semi structured proforma.

b) Patient's records and registered.

c) High power X-ray machines $(300 \mathrm{~mA}, 500 \mathrm{~mA}$ and $800 \mathrm{~mA})$.

d) High frequency USG machine with 5-12 MHZ linear transducer in HD-7 model M/S PHILIPS makes.

e) CT scan machine: M/S HITACHI make PRONTO model, single slice spiral CT scan.

f) MRI machine: 1.5 Tesla, M/S GE make, Bravo model.

\section{RESULTS}

In this cross sectional descriptive study, total 56 numbers of patients with clinically diagnosed knee $\mathrm{OA}$ were studied, in the department of Radiodiagnosis, North Bengal Medical College and Hospital, Darjeeling. All patients were assessed by history taking, clinical examination followed by MRI, conventional radiography, USG and CT-Scan examination. Data of different variables in different modalities were collected. The final analysis for all the variables was done by standard statistical software and results were as follows:

Demographic profile and clinical findings of the patients

The mean age of the patients was 61.13 years. The number of male and female patients was 18 and 38 respectively. 
The TF compartment was more frequently and severely affected in OA of knee joint than the patellofemoral (PF) compartment and within TF compartment, medial TF (MTF) compartment was affected more frequently. Bone marrow lesion (BML) was found among $51.78 \%$ of patients in this study, and the most common site of BML was in MTF compartment (68.96\%). Cruciate ligament abnormality was seen in 13 (23.21\%) of study population, among which anterior cruciate ligament abnormality was seen more frequently $12.5 \%$ cases and posterior cruciate ligament in $10.7 \%$ cases. Collateral ligament involvement was seen rarely, only in two $(3.56 \%)$ patients out of 56 patients in this study. Meniscal abnormality in the form of degeneration was found in $21(37.5 \%)$ patients and among these patients medial meniscus was involved in $12(21.42 \%)$ patients and lateral meniscus was involved in $9(16.07 \%)$ patients. Osteophyte was detected in $92.85 \%$ patients with conventional radiography but in 100\% patients with CT scan and MRI. In all modality in our study, most frequent site of osteophytosis was MTF compartment. In $85.71 \%, 96.42 \%$ and $96.42 \%$ of patient, osteophytes was identified in MTF compartment in conventional radiography, CT scan and MRI respectively.

Comparison of USG, CT scan and MRI imaging findings The imaging findings studied in the patients were JSN, meniscal abnormality, Baker's cyst, cruciate ligament

\begin{tabular}{|c|c|c|c|}
\hline Imaging finding & Radiography & CT scan & $P$ value \\
\hline Joint space narrowing & $36 / 56$ & $28 / 56$ & 0.12 \\
\hline Sub-chondral cyst & $24 / 56$ & $36 / 56$ & 0.018 \\
\hline Loose bodies & $08 / 56$ & $21 / 56$ & 0.004 \\
\hline
\end{tabular}

\begin{tabular}{|c|c|c|c|}
\hline & & $M P I$ & P ylu \\
\hline Knee effusion & $44 / 56$ & $48 / 56$ & 0.22 \\
\hline Synovial thickening & $37 / 56$ & $44 / 56$ & 0.10 \\
\hline
\end{tabular}

\begin{tabular}{|c|c|c|c|}
\hline Imaging finding & CT Scan & MRI & $P$ value \\
\hline $\begin{array}{l}\text { Sub-chondral cyst } \\
\text { Loose bodies }\end{array}$ & $\begin{array}{l}36 / 56 \\
21 / 56\end{array}$ & $\begin{array}{l}36 / 56 \\
15 / 56\end{array}$ & $\begin{array}{l}1.00 \\
0.22\end{array}$ \\
\hline
\end{tabular}

abnormality, knee effusion, subchondral cyst and loose bodies. A comparison between radiography and CT scan was done for JSN, subchondral cyst and loose bodies (Table 1). Similarly, a comparison was done between USG and MRI for detection of knee effusion and synovial thickening (Table 2). Finally, CT scan and MRI were compared for the detection of subchondral cysts and loose bodies (Table 3).

\section{DISCUSSION}

Early diagnosis and specific measures is the only way to reduce disability from this crippling disease. ${ }^{5} \mathrm{MRI}$, though very sensitive for early detection of $\mathrm{OA}$, cost is limiting factor. In other modality like conventional radiography, high frequency USG and CT scan, though cost is low, but all the early osteoarthritic changes are not detected by any of this modality alone. ${ }^{6}$

This cross-sectional observational study was undertaken to evaluate knee OA with MRI and then compare those findings with conventional radiography, CT scan and high frequency USG findings.

Fifty six patients were included in the study and their detailed demographic, clinical and imaging parameters were assessed. Maximum numbers of patients were female and maximum numbers of study population were above $60 \mathrm{yrs}$ of age. All the patients were having symptomatic knee OA. MTF compartment was most frequently affected among three knee compartments. Only MRI detectable changes were cartilaginous defect, BML, meniscal abnormality and cruciate ligament changes in addition to other changes detectable by other modalities.

Cartilage change was seen in approximately one-third case. BML, JSN, osteophytosis, subchondral sclerosis, and subchondral cyst were predominantly seen in MTF compartment.

Significant difference was seen between conventional radiography and CT scan in the detection of loose bodies and subchondral cyst (P-value 0.004 and 0.018 respectively). Loose body and subchondral cyst was better detected on CT scan than radiograph. Detection rate of subchondral cyst on CT scan and on MRI is equal, so relation of Radiography with MRI in detection of subchondral cyst is similar to relation with CT scan, which is significant.

No significant difference was found between conventional radiography and CT scan for detection of JSN and osteophytes (P-value 0.09 and 0.06 respectively). Osteophytes in the different compartments of the knee joint were detected 
both on CT scan and MRI in similar frequency. Subchondral sclerosis was equally detected on conventional radiography, CT scan, and MRI. Moreover, no significant difference was found between high frequency USG and MRI for the detection of joint effusion, synovial thickening (P-value 0.22 and 0.10 respectively). High frequency USG and MRI were almost equally effective for detection of Baker's cyst.

The present study was carried out over a period of 1 year on 56 numbers of patients with clinically diagnosed knee OA, in the Department of Radiodiagnosis, NBMCH, Darjeeling with the aim to find out the osteoarthritic changes of knee by MRI and compare those findings with conventional radiography, high frequency USG and CT scan findings.

In our study, the TF compartment was more frequently and severely affected in $\mathrm{OA}$ of knee joint than the PF compartment and within TF compartment, MTF compartment was affected more frequently.

In this study, MRI was a unique modality for detection of cartilage defect, BML, meniscal lesion, ligamentous lesion; no other modality could detect the changes in $\mathrm{OA}$ of the mentioned structures.

In the present study, articular cartilage defect on MRI was found in $41(73.21 \%)$ patients, while in a study Joshi et al., found articular cartilage defect in almost every patient. Foo et al., in their study found articular cartilage defect in $37.1 \%$ of the patients. ${ }^{7}$ However Bruyère et al., in their study reported articular cartilage defect in $75 \%$ patients, which is very close to our study result. ${ }^{8}$

BML was found among $51.78 \%$ of patients in this study and the most common site of BML was in MTF compartment $(68.96 \%)$. Bruyère et al., in their study reported BML in $57 \%$ patients. ${ }^{8}$ Bacon et al. in their study found BML in $46.9 \%$ of knee joint and Fernandez-Madrid et al., found BML in $65 \%$ of the patients. ${ }^{9}$

Meniscal abnormality in the form of degeneration was found in $21(37.5 \%)$ patients and among these patients medial meniscus was involved in $12(21.42 \%)$ patients and lateral meniscus was involved in $9(16.07 \%)$ patients. Bruyère et al., in their study found meniscal abnormality in $27 \%$ of the study population. ${ }^{8}$

Cruciate ligament abnormality was seen in $13(23.21 \%)$ of study population, among which anterior cruciate ligament abnormality was seen more frequently $12.5 \%$ cases and posterior cruciate ligament in $10.7 \%$ cases. Collateral ligament involvement was seen rarely, only in two $(3.56 \%)$ patients out of 56 patients in this study. Joshi et al., was found ACL and PCL involvement in $25 \%$ and $34.7 \%$ respectively. No tendon abnormality was found in any of the study population.

JSN was found in $64.28 \%$ and $50 \%$ patients in conventional radiography and in CT scan respectively and in both of this study, MTF compartment involvement was seen more frequently. The probable reason behind it is that JSN on conventional radiography is seen in weight bearing $\mathrm{AP}$ view, in which very minute amount narrowing can be detected accurately, where as on CT scan, JSN is seen in scanogram or in reformatted coronal image, which is taken in supine position; so JSN could not be measured accurately. ${ }^{10}$

Osteophyte was detected in $92.85 \%$ patients with conventional radiography but in $100 \%$ patients with CT scan and MRI. In all modality in our study, most frequent site of osteophytosis was MTF compartment. In $85.71 \%, 96.42 \%$ and $96.42 \%$ of patient, osteophytes was identified in MTF compartment in conventional radiography, $\mathrm{CT}$ scan and MRI respectively. Ai et al., reported osteophytes in $100 \%$ of the knee affected by OA on MRI. ${ }^{11}$ Chan et al., in their study found that in the medial compartment CT and MRI showed osteophytes in 100\% of the knee where as radiography showed osteophytes in only $60 \%$ of the cases. ${ }^{12}$ In this study, no significant difference was found between CT scan and MRI in the detection of osteophytes. So, for only detection of osteophyte CT scan and MRI will be supplementary to each other.

Subchondral sclerosis was equally detected on conventional radiography, CT scan, and MRI and it was seen in $71.42 \%$ of study population and predominantly seen in MTF compartment. Bruyère et al., reported subchondral sclerosis in $43 \%$ of patients. ${ }^{8}$ So, conventional radiography will be supplementary to CT scan and MRI for the detection of subchondral sclerosis. ${ }^{13}$

Subchondral cyst was found in 24(42.85\%), $36(64.28 \%)$ and $36(64.28 \%)$ of the study population on conventional radiography, CT scan and MRI respectively and with all modality subchondral cyst was maximally detected in MTF compartment. Wu et al., in their study found subchondral cysts in $50 \%$ of patients on MRI. ${ }^{14}$ In this present study, no difference was seen between CT scan and MRI in the detection of subchondral cyst but significant difference was seen between conventional radiography and CT scan, and conventional radiography and MRI. Low detection rate of the subchondral cyst on conventional radiography is possibly due to obscuration of small cyst in deeper part of condyles by the impression of overlapping thick bone on two dimensional radiographic film. ${ }^{15}$

Loose body was found in 21(37.5\%), 15(26.78\%) and 8 (14.28\%) of patients on CT scan, MRI and conventional radiography respectively. There was a significant difference 
between CT scan and two other modalities like MRI and conventional radiography, in the detection of loose body. In this study, CT scan was the best modality for the detection of loose bodies.

Knee effusion was seen on USG and MRI in $78.57 \%$ and $85.71 \%$ of study population respectively. FernandezMadrid et al., in their study found knee effusion in $60 \%$ of patients on MRI. ${ }^{16}$ Tarhan and Unlu in a study reported knee effusion in $70 \%$ and $85 \%$ of patients on USG and MRI respectively, which is almost similar to the result of the present study. ${ }^{17}$

In this study, synovial thickening was detected more commonly on MRI than USG and on USG and MRI synovial thickening was seen in $66.07 \%$ and $78.57 \%$ of the cases respectively. This difference was probably due to the fact that, on MRI synovial thickening was seen in multiple locations where as on USG synovial thickening was seen only in suprapatellar pouch region; ${ }^{18}$ So, on USG we may missed the synovial thickening in the region other than suprapatellar pouch of knee, which are easily seen on MRI. Tarhan-Unlu et al., reported in their study that, synovial thickening was seen in $73 \%$ case of study population on MRI. ${ }^{17}$ Tarhan and Unlu in their study found synovial thickening in 34\% and $50 \%$ of cases on USG and MRI respectively and MRI was superior modality than USG in the detection of synovial thickening, similar to this present study.

Baker's cyst was seen in $25(44.64 \%)$ cases of study population and the incidence of detection of Baker's cyst with USG and MRI were same. Hall et al., in their study found baker's cyst in 39\% of study population which is nearer to this study result. ${ }^{19}$ Joshi et al., found baker cyst in $40.63 \%$ of the patients. ${ }^{20}$ Where as Tarhan and Unlu found baker cyst in $40 \%$ and $35 \%$ of cases on USG and MRI respectively. ${ }^{17}$

\section{Limitations of the study}

- In this study, the cartilage thickness measurement using high frequency USG was not done in our study set-up, due some technical problem.

- In this study cartilage changes on MRI was assessed only qualitatively, no quantitative assessment was done.

- In this study non contrast MRI of knee was done, no contrast enhanced MRI done, so there is chance of missing actual number of case even with minimal synovial thickening.

\section{CONCLUSION}

MRI is unique modality for detection of articular cartilage destruction, even if it is very minimal (earliest change),
BML, meniscal abnormality and cruciate ligament abnormality. However, in this present study, among those MRI findings which are comparable with the findings of other three modalities, MRI was seen superior to conventional radiography for the detection of subchondral cyst but in that respect it was seen equally effective with CT scan. So, though MRI is costly, not always available and not feasible for every patient, in this modern era, when disease modifying drugs are available and early diagnosis may change the disease progression, MRI is the best modality for early detection of OA changes of the knee joint. However, for follow-up MRI or any one of the other three modalities can be used alone or in combination, which will detect almost all changes of advanced $\mathrm{OA}$ and will reduce cost to the patients and will be readily available to every patient.

\section{ACKNOWLEDGMENT}

The authors acknowledge the help and support of the staff and faculty of Radiology Department of North Bengal Medical College \& Hospital, West Bengal, India.

\section{REFERENCES}

1. Pal CP, Singh P, Chaturvedi S, Pruthi KK and Vij A. Epidemiology of knee osteoarthritis in India and related factors. Indian J Orthop. 2016;50(5):518-522.

https://doi.org/10.4103/0019-5413.189608

2. Lespasio MJ, Piuzzi NS, Husni ME, Muschler GF, Guarino A and Mont MA. Knee osteoarthritis: A primer. Perm J. 2017;21:16-183.

3. Oo WM, Linklater JM and Hunter DJ. Imaging in knee osteoarthritis. Curr Opin Rheumatol. 2017;29(1):86-95.

4. Hayashi D and Roemer FW, Guermazi A. Imaging for osteoarthritis. Ann Phys Rehabil Med. 2016;59(3):161-169.

5. Wang L, Chen Q, Tong P, Xia L and Jin H. Progress on the early diagnosis of knee osteoarthritis. Zhongguo Gu Shang (China J Orthop Traumatol). 2016;29(3):288-291.

6. Boegård $T$ and Jonsson $K$. Hip and knee osteoarthritis. Conventional $\mathrm{X}$-ray best and cheapest diagnostic method. Lakartidningen. 2002;99(44):4358-4360.

7. Potter HG and Foo LF. Magnetic resonance imaging of articular cartilage: Trauma, degeneration, and repair. Am J Sports Med. 2006;34(4):661-677.

https://doi.org/10.1177/0363546505281938

8. Bruyère $\mathrm{O}$, Honvo $\mathrm{G}$, Veronese $\mathrm{N}$, Arden NK, Branco J, Curtis EM, et al. An updated algorithm recommendation for the management of knee osteoarthritis from the European society for clinical and economic aspects of osteoporosis, osteoarthritis and musculoskeletal diseases (ESCEO). Semin Arthritis Rheum. 2019;49(3):337-350.

https://doi.org/10.1016/j.semarthrit.2019.04.008

9. Bacon K, LaValley MP, Jafarzadeh SR and Felson D. Does cartilage loss cause pain in osteoarthritis and if so, how much? Ann Rheum Dis. 2020;79(8):1105-1110.

http://doi.org/10.1136/annrheumdis-2020-217363

https://doi.org/10.1136/annrheumdis-2020-218178 
10. Suzuki N and Seino K. Quantitative analysis for narrowing of radiographic joint space in knee osteoarthritis. Nihon Hoshasen Gijutsu Gakkai Zasshi. 2013;69(3):251-256.

https://doi.org/10.6009/jjt.2013_jsrt_69.3.251

11. Ai F, Yu C, Zhang W, Morelli JN, Kacher D and Li X. MR imaging of knee osteoarthritis and correlation of findings with reported patient pain. J Huazhong Univ Sci Technolog Med Sci. 2010;30(2):248-254.

https://doi.org/10.1007/s11596-010-0223-0

12. Chan WP, Lang P, Stevens MP, Sack K, Majumdar S, Stoller DW, et al. Osteoarthritis of the knee: Comparison of radiography, CT, and MR imaging to assess extent and severity. AJR Am J Roentgenol. 1991;157(4):799-806. https://doi.org/10.2214/ajr.157.4.1892040

13. Buckland-Wright $C$. Subchondral bone changes in hand and knee osteoarthritis detected by radiography. Osteoarthritis Cartilage. 2004;12 Suppl A: S10-S19. https://doi.org/10.1016/j.joca.2003.09.007

14. Wu H, Webber C, Fuentes CO, Bensen R, Beattie K, Adachi JD, et al. Prevalence of knee abnormalities in patients with osteoarthritis and anterior cruciate ligament injury identified with peripheral magnetic resonance imaging: A pilot study. Can Assoc Radiol J. 2007;58(3):167-175.

https://doi.org/10.1016/s1063-4584(07)61747-6

15. Bousson V, Lowitz T, Laouisset L, Engelke K and Laredo JD.
CT imaging for the investigation of subchondral bone in knee osteoarthritis. Osteoporos Int J. 2012;23 Suppl 8:S861-S865. https://doi.org/10.1007/s00198-012-2169-5

16. Fernandez-Madrid F, Karvonen RL, Teitge RA, Miller PR and Negendank WG. MR features of osteoarthritis of the knee. Magn Reson Imaging. 1994;12(5):703-709. https://doi.org/10.1016/0730-725x(94)92194-6

17. Tarhan $S$ and Unlu $Z$. Magnetic resonance imaging and ultrasonographic evaluation of the patients with knee osteoarthritis: A comparative study. Clin Rheumatol. 2003;22(3):181-188. https://doi.org/10.1007/s10067-002-0694-x

18. Mathiessen A and Conaghan PG. Synovitis in osteoarthritis: Current understanding with therapeutic implications. Arthritis Res Ther. 2017;19(1):18. https://doi.org/10.1186/s13075-017-1229-9

19. Hall M, Doherty S, Courtney P, Latief K, Zhang W and Doherty M. Synovial pathology detected on ultrasound correlates with the severity of radiographic knee osteoarthritis more than with symptoms. Osteoarthritis Cartilage. 2014;22(10):1627-1633. https://doi.org/10.1016/j.joca.2014.05.025

20. Joshi AD, Gupta S, Gaur AK, Mhambre AS and Shukla A. Demographic profile and spectrum of ultrasonographic findings in medial knee pain: An observational study. Indian J Med Sci. 2020;72(3):201-205.

https://doi.org/10.25259/ijms_44_2020

\section{Authors Contribution:}

RS- Concept and design of the study, prepared first draft of manuscript; CKD- Interpreted the results; reviewed the literature and manuscript preparation; DS- Concept, coordination, statistical analysis and interpretation, preparation of manuscript and revision of the manuscript

Work attributed to:

North Bengal Medical College and Hospital, West Bengal, India

Orcid ID:

Dr. Raju Singha - (D) https://orcid.org/0000-0002-4070-2141

Dr. Chanchal Kumar Dalai - (1) https://orcid.org/0000-0002-5406-3440

Dr. Deblina Sarkar - (1) https://orcid.org/0000-0002-2294-5078

Source of Funding: None, Conflicts of Interest: None. 